\title{
Educación financiera en estudiantes universitarios
}

\section{Financial education in university students}

\author{
DOI: http://dx.doi.org/10.179 81/econcuc.38.2.2017.08
}

Recibido: may 19/2017 Devuelto para revisión: sep 06/17 Aceptado: oct 25/2017

Leonardo Beltrán Pinto ${ }^{1}$

Efraín Gómez Martínez ${ }^{2}$

\begin{abstract}
Resumen
La buena gestión financiera repercute en el bienestar sostenido de las personas, por lo cual se hace necesario contar con competencias financieras idóneas para tal fin. Utilizando el test Chi-Cuadrado (X2), el presente estudio analizó en 200 estudiantes universitarios de grados medios (V y VI semestre), el nivel de educación financiera y su relación con otras variables socioeconómicas. Se encontró que no existían relaciones significativas entre ellas, salvo con la edad. No se halló relación entre el nivel de educación financiera y estar estudiando un programa asociado a las ciencias económicas.
\end{abstract}

Palabras clave: bienestar, educación financiera, entorno, conductas adecuadas, Chi-Cuadrado.

\begin{abstract}
The financial management affects the well-being sustained people, making it necessary to have financial skills suitable for this purpose. Using the Chi-square test (X 2), this study analyzed 200 University students of middle grades (V and VI semester), the level of financial education and its relationship with other socio-economic variables. It was found that significant relationships between them, there were no except with age. No relationship was found between the level of financial literacy and be studying a program associated with the economics.
\end{abstract}

Keywords: welfare, Financial education, environment, appropriate behaviors, Chi-Square.

Clasificación JEL: D10, D19, D69, G29

\footnotetext{
${ }^{1}$ Administrador de Empresas, MBA mención Gerencia Financiera, Magíster en Desarrollo Social, Máster en Proyectos Internacionales, Docente tiempo completo Corporación Universitaria del Caribe.

${ }^{2}$ Magister en Administración de Empresas, Especialista en Administración Financiera. Docente tiempo completo de la Corporación Universitaria del Caribe.
}

The author; licensee Universidad de la Costa - CUC 


\section{Introducción}

La globalización de los mercados y el avance en la tecnología ha permitido la continua innovación de los productos financieros. A partir del comienzo de la vida laboral, los trabajadores colombianos se les permite el acceso a diferentes alternativas de ahorros y tipos de créditos, lo que representa una oportunidad. Sin embargo, este se puede convertir en un problema para estos, por la escasa oportunidades de formación financiera con la que cuentan. La Organisation for Economic Co-operation and Development-OCDE (2005a) enumera una serie de problemas que se presentan por el desconocimiento de los productos financieros como lo son:

a. El incremento de los aportes de los estados a las pensiones por parte del desconocimiento de los trabajadores en los fondos de retiros pensionales;

b. El crecimiento en la deuda de los trabajadores a niveles históricos y como resultado, muchos jóvenes han sido cargados con sobreendeudamientos cuando tratan de comprar una casa e iniciar una familia;

c. Existe un porcentaje aún bastante considerable que aún no participa del sistema financiero, principalmente por el desconocimiento de este.

Con el objetivo del ingreso de Colombia a la OCDE, el país participa en las pruebas PISA ${ }^{3}$ que evalúa las capacidades que tienen los alumnos de $18^{4}$ países. Esta prueba permite realizar un análisis comparativo de la formación percibida por los alumnos colombianos con respecto a los países industrializados. En dichas pruebas fueron evaluados aspectos relacionados con la lectura, la matemática y la

\footnotetext{
${ }^{3}$ Programa para la Evaluación Internacional de Alumnos (PISA, por sus siglas en inglés)

${ }^{4}$ Los 13 miembros de la OCDE para el año 2012 fueron: Australia, Bélgica, Eslovaquia, Eslovenia, España, Estados Unidos, Estonia, Francia, Israel, Italia, Nueva Zelanda, Polonia y República Checa, y 5 países asociados: Colombia (ingresó año 2018), Croacia, Federación Rusa, Letonia y Shanghai-China.
}

ciencia, pero a partir del año 2012, la educación financiera entra como nuevo componente que sirve para evaluar la capacidad de los estudiantes para tomar decisiones financieras de la vida cotidiana y la planeación del futuro. Los resultados con respecto a este último componente, no fueron los mejores, los estudiantes colombianos ocuparon el último lugar entre los países evaluados; sin embargo, en este país hasta hace poco se está implementando la educación financiera como política de gobierno para la pre-adolescencia lo que pudiera mejorar ese resultado.

En Colombia se reglamenta la necesidad de formación en educación financiera a partir de la ley 1328 de 2009 que señala que,

"Las entidades vigiladas (bancos), las asociaciones gremiales, las asociaciones de consumidores, las instituciones públicas que realizan la intervención y supervisión en el sector financiero, así como los organismos de autorregulación, procurarán una adecuada educación de los consumidores financieros ${ }^{5}$ respecto de los productos y servicios financieros que ofrecen las entidades vigiladas, de la naturaleza de los mercados en los que actúan, de las instituciones autorizadas para prestarlos, así como de los diferentes mecanismos establecidos para la defensa de sus derechos" (Congreso de Colombia, 2009, Art. 3. Paragrafo (f)).

Por lo anterior, el Congreso de la República resalta que la educación ${ }^{6}$ financiera no solo es para los inversionistas o clientes, sino que también para todo aquel que puede acceder al sistema financiero por lo cual "consolidó una alianza público-privada, formalizada en el convenio 024 del 2012, para el diseño e implementación de un programa que cumpliera con este mandato. El 3 de julio del mismo año se presentó el primer gran resultado de este convenio: el documento que contiene las orientaciones pedagógicas para la educación

\footnotetext{
${ }^{5}$ Consumidor financiero: Es todo cliente, usuario o cliente potencial de las entidades vigiladas.

${ }^{6}$ Es definida como la comunicación organizada y sustentada, que está diseñada para producir aprendizaje (OCDE, 2004).
} 
económica y financiera para ser impartida desde primero hasta el onceavo grado" (ASOBANCARIA, 2014).

Lo anterior, denota los esfuerzos institucionales por la consecución de la alfabetización financiera. Esperando así, que los consumidores financieros puedan tomar decisiones activas, participativas, críticas y reflexivas, con capacidades de interpretar los eventos que puedan afectarlos directa o indirectamente y mejorar las competencias para tomar decisiones personales y sociales sobre la multitud de problemas económicos que se encuentran en la vida cotidiana (Denegrí, Del Valle, González, Etchebar-ne, Sepúlveda, \& Sandoval, 2014). Por lo cual, esta investigación tiene como objetivo analizar la educación financiera de los jóvenes estudiantes de los diferentes programas de CECAR con relación a su formación, edad, nivel socioeconómico.

\section{Marco referencial}

Existen diferentes conceptos del significado de la educación financiera. Según la Organización para la Cooperación y el Desarrollo Económico (OCDE) se definida como:

"El proceso por el que los consumidores financieros/inversores mejoran su comprensión de los productos financieros, conceptos y riesgos y, a través de la información, la enseñanza y/o el asesoramiento objetivo, desarrollan las habilidades y confianza para adquirir mayor conciencia de los riesgos y oportunidades financieras, tomar decisiones informadas, saber dónde acudir para pedir ayuda y tomar cualquier acción eficaz para mejorar su bienestar financiero" (OCDE, 2005b)

Desde el concepto de la OCDE, se plantea como hecho fundamental que los consumidores financieros, puedan comprender los conceptos, riesgos y oportunidades asociados a la adquisición de productos financieros, además de desarrollar habilidades para tomar decisiones informadas. Ahora bien, la importancia de la educación financiera esta soportada por las recientes investigación asociadas a la crisis financiera del (2008) en Estados Unidos, autores como Gerardi, Götte, Meier, Goette, \& Meier (2010) demuestran a través de evidencia empírica que los riesgos incumpliendo en los pagos son más altos para las instituciones financiera que otorgaron créditos a personas que cuentan con una deficiente conocimiento financiero, por lo que otorgar créditos a este tipo de personas pudo ser un factor que agravara la crisis.

Del mismo modo, otro autores como Bernheim \& Scholz (1993) señalan que las personas sin educación universitaria ahorran muy poco y al momento de pensionarse los hogares se ven obligados a reducir su estándar de vida, en contraste, las personas que tienen acceso a educación universitaria ahorran más para la jubilación y planifican mejor gastos. Así mismo, autores como Lusardi \& Tufano (2015) y Klot, Peters, Aalto, Bellander, Berglind, D’Ippoliti, Elosua, Hörmann, Kulmala, Lanki, Löwel, Pekkanen, Picciotto, Sunyer, $\&$ Forastiere, 2005) indican que existe una relación directa en el sobre endeudamiento de los hogares y el analfabetismo financiero y resaltan, que las personas con poco conocimiento financiero adquieren créditos con altas tasas de interés y que lo más común es que estas personas provengan de los grupos económicos más vulnerables.

En cuanto a la correlación por sexo, la literatura demuestra que son las mujeres son quienes tienen menos conocimiento financiero, además señalan que la edad es otra variable que influye en el buen comportamiento financiero, pues son las personas de mayor edad las más vulnerables, estas personas presentan poco conocimientos de factores macroeconómicos como la inflación, además de presentar poco conocimiento de planificación financiera y diversificación de riesgo (Lusardi \& Mitchell, 2011). Por consiguiente, la literatura demuestra a través de evidencia empírica que la alfabetización financiera puede incentivar el ahorro de la población, además de reducir los riesgos asociados a los incumplimientos de pagos y contribuir a la normalización de los créditos. 


\section{Diseño Metodológico}

El presente estudio basa su análisis en un enfoque cuantitativo, que representa un proceso sistemático, secuencial, de cara a su desarrollo (Hernández, Fernández y Baptiste, 2014). Estas cualidades se materializan en la disposición de los autores de estudiar las posibles relaciones intervariables del nivel de Educación Financiera (EF), estructurado de acuerdo a los modelos estadísticos explicados más adelante. En cuanto al diseño específico del estudio, es no experimental, de corte descriptivo y correlacional. Descriptivo, pues busca detallar la EF como una categoría conceptual que precisa conductas humanas pro futuro económico estable, poseedora de características propias. Correlacional, en cuanto presume la EF como una variable dependiente, cuya variabilidad estaría asociada a otras variables inherentes al ser humano, como el nivel educativo, el contexto socioeconómico, la edad o la orientación vocacional.

Viéndolo en profundidad, este estudio pretende estudiar preliminarmente el nivel de EF en una muestra de estudiantes universitarios, y en lo sucesivo, generar insumos referenciales para futuros estudios relacionados con mayor complejidad a nivel de expansión poblacional.

A nivel metodológico, se inspecciona el nivel de EF frente a la edad de los estudiantes, su estrato social, el programa académico en curso y el sexo. En principio, se muestra un análisis descriptivo de las frecuencias, relativas y absolutas de cada variable, con la intención de dilucidar el grado participativo de cada una de ellas dentro del estudio. Posteriormente se muestran los resultados de correlación entre las variables objeto. Para tal fin, se usó la Prueba Chi-Cuadrado de Pearson, al ser la herramienta estadística que facilita el estudio relacional entre variables de diferentes tipos, especialmente cuando entre ellas interactúa alguna de carácter cualitativo. Para el análisis básico inicial, se construyeron tablas de frecuencia estadística; para la inspección de hipótesis, se utilizó el software IBM SPSS en la entrada de cálculo "Chi Cuadrado".
No obstante, en lo referente al análisis de relación entre el nivel de EF y la variable "programa cursado", se realizó una inspección ampliada, siguiendo el modelo Chi Cuadrado desde la especificidad de Tablas de Contingencia. En la tabla 1 se ilustran las variables usadas y sus identificadores de entrada para el software.

\section{Hipótesis}

De acuerdo a la revisión bibliográfica, la variable "nivel de $E F$ " guarda relación con otras variables asociadas con la condición humana y su conexión con el medio que le rodea. En tal sentido, se presentaron las siguientes hipótesis " $H$ ", cada una vinculada a las variables consideradas independientes o de referenciación; en principio todas las restantes. $H_{0}$ relaciona hipótesis de partida o nulas, las cuales consideran independencia entre variables. $H_{i}$ ilustra las alternativas a la nulidad, es decir, es la hipótesis que denota relación. Las consideraciones hipotéticas se constituyeron así:

\section{H10: $\quad$ El nivel de EF es independiente al programa cursado}

H1: $\quad$ El nivel de EF está relacionado a la programa cursado

H2, $\quad$ El nivel de EF es independiente a la edad del estudiante

$\mathrm{H}_{i}:$ : El nivel de EF está relacionado a la edad del estudiante

$H 3_{0}$ : $\quad$ El nivel de EF es independiente al estrato social del estudiante

$H 3_{i}$ : $\quad$ El nivel de EF está relacionado al estrato social del estudiante

H4: $\quad$ El nivel de EF es independiente al sexo del estudiante

$\mathrm{H}_{i}$ : $\quad$ El nivel de EF está relacionado al sexo del estudiante

Para la inspección de hipótesis se asume una confianza del $95 \%$ y alfa de error máximo admisible de $5 \%$. 
Tabla 1

Detalle de variables analizadas

\begin{tabular}{|c|c|c|c|}
\hline $\begin{array}{c}\text { ID de } \\
\text { variable }\end{array}$ & $\begin{array}{l}\text { Tipo de } \\
\text { variable }\end{array}$ & Descripción de variable & Categorías \\
\hline $\begin{array}{l}\mathrm{N}^{\circ} \\
\text { ESTUDI }\end{array}$ & Discreta & $\begin{array}{l}\text { Categoriza a cada encuestado con un número } \\
\text { de identificación }\end{array}$ & No aplica \\
\hline \multirow{3}{*}{$\begin{array}{l}\text { NIVEL }_{-} \\
\text {EDUFIN }\end{array}$} & \multirow{3}{*}{ Ordinal } & \multirow{3}{*}{$\begin{array}{l}\text { Representa el nivel de educación financiera de } \\
\text { cada individuo }\end{array}$} & Alto \\
\hline & & & Medio \\
\hline & & & Bajo \\
\hline \multirow{2}{*}{$\begin{array}{l}\text { SEME } \\
\text { ACADEM }\end{array}$} & \multirow{2}{*}{ Ordinal } & \multirow{2}{*}{ Semestre académico cursado } & Quinto \\
\hline & & & Sexto \\
\hline \multirow{10}{*}{$\begin{array}{l}\text { PROG }_{-} \\
\text {ACADEM }\end{array}$} & \multirow{10}{*}{ Nominal } & \multirow{10}{*}{$\begin{array}{l}\text { Programa académico estudiado según lo } \\
\text { estipulado en el diseño de la muestra }\end{array}$} & DERECHO \\
\hline & & & PSICOLOGÍA \\
\hline & & & ING. SISTEMAS \\
\hline & & & ARQUITECTURA \\
\hline & & & C. DEPORTE \\
\hline & & & $\begin{array}{l}\text { ING. } \\
\text { INDUSTRIAL }\end{array}$ \\
\hline & & & T. SOCIAL \\
\hline & & & ADMON \\
\hline & & & CONTADURÍA \\
\hline & & & ECONOMÍA \\
\hline \multirow{2}{*}{$\begin{array}{l}\text { CIENCIA } \\
\text { ASOCIA }\end{array}$} & \multirow{2}{*}{ Nominal } & \multirow{2}{*}{$\begin{array}{l}\text { Agrupa a los programas académicos en estas } \\
\text { dos categorías, por su relación con la EF. }\end{array}$} & $\begin{array}{l}\text { NO } \\
\text { ECONÓMICAS }\end{array}$ \\
\hline & & & ECONÓMICAS \\
\hline $\begin{array}{l}\text { EDAD } \\
\text { AÑOS }\end{array}$ & Continua & Edad de cada encuestado & No Aplica \\
\hline \multirow{7}{*}{$\begin{array}{l}\text { ESTRA- } \\
\text { SOCIAL }\end{array}$} & \multirow{7}{*}{ Ordinal } & \multirow{7}{*}{$\begin{array}{l}\text { Nivel socioeconómico según estratificación } \\
\text { colombiana }\end{array}$} & CERO \\
\hline & & & UNO \\
\hline & & & DOS \\
\hline & & & TRES \\
\hline & & & CUATRO \\
\hline & & & CINCO \\
\hline & & & SEIS \\
\hline \multirow{2}{*}{ SEXO_H_M } & \multirow{2}{*}{ Nominal } & \multirow{2}{*}{ Sexo natural: hombre o mujer } & HOMBRE \\
\hline & & & MUJER \\
\hline
\end{tabular}

Fuente: elaboración propia. 


\section{Diseño de la muestra}

La población objeto de estudio comprende estudiantes de pregrado, de los doce programas de modalidad presencial, que forman parte de la Corporación Universitaria del Caribe (CECAR), de los diez y ocho años de edad en adelante, y que no sean casados o padres de familia; debido a que este sector de la población es atendido en temas de educación por parte de las entidades bancarias y de crédito, quienes, por ley, les brindan asesorías y programas de educación financiera acerca de sus productos.

El muestreo fue de tipo "no probabilístico" debido a que no fue utilizado el algoritmo para la determinación de la muestra. En las muestras no probabilísticas, la elección de los elementos no depende de la probabilidad, sino de causas relacionadas con las características de la investigación o los propósitos del investigador (Hernández, Fernández y Baptiste, 2014). Fueron seleccionados aleatoriamente 20 estudiantes de los grados V y VI en cada programa académico de la modalidad presencial. Excluyendo a los grados inferiores y superiores, con la intención de tener una base muestral lo más homogénea posible, en lo que a nivel de formación respecta. El proceso fue selectivo, al excluir sujetos casados o padres de familia en la muestra.

\section{Instrumento}

Con base en trabajos anteriores desarrollados por la Organización para la Cooperación y el Desarrollo Económico-OCDE, se ajustó un instrumento homólogo al ya estructurado y validado por dicha organización; el cual se trata de una encuesta de medición de capacidades financieras "International Survey of financial literacy for adults". Dicha encuesta está estructurada en tres componentes generales: 1. Conocimiento financiero, 2. Comportamiento financiero y 3. Actitud financiera. Entre ellos se distribuyen 17 preguntas específicas, de respuestas seleccionadas y tipología nominal; con componentes que tienen una valoración media, ponderada por el número de preguntas que conforman cada uno. Se entienden en cada componente, preguntas asertivas y otras negativas, frente al ideal conductual y cognoscitivo referenciado en la revisión bibliográfica.

Tabla 2

Medidas de tendencia central "EDAD"

\begin{tabular}{|c|c|c|c|}
\hline EDAD_AÑOS & \multicolumn{3}{|c|}{ Medidas de frecuencia } \\
\hline MEDIA & \multicolumn{3}{|c|}{18,56} \\
\hline MEDIANA & \multicolumn{3}{|c|}{19} \\
\hline MODA & \multicolumn{3}{|c|}{19} \\
\hline MAX & \multicolumn{3}{|c|}{20} \\
\hline MIN & \multicolumn{3}{|c|}{17} \\
\hline RANGO INERCUARTIL & \multicolumn{3}{|c|}{1} \\
\hline VALORES DISTINTOS & Edades & $f i$ & $h i(\%)$ \\
\hline \multirow{4}{*}{4} & 17 & 36 & $18,0 \%$ \\
\hline & 18 & 54 & $27,0 \%$ \\
\hline & 19 & 72 & $36,0 \%$ \\
\hline & 20 & 38 & $19,0 \%$ \\
\hline TOTAL & & 200 & $100 \%$ \\
\hline
\end{tabular}

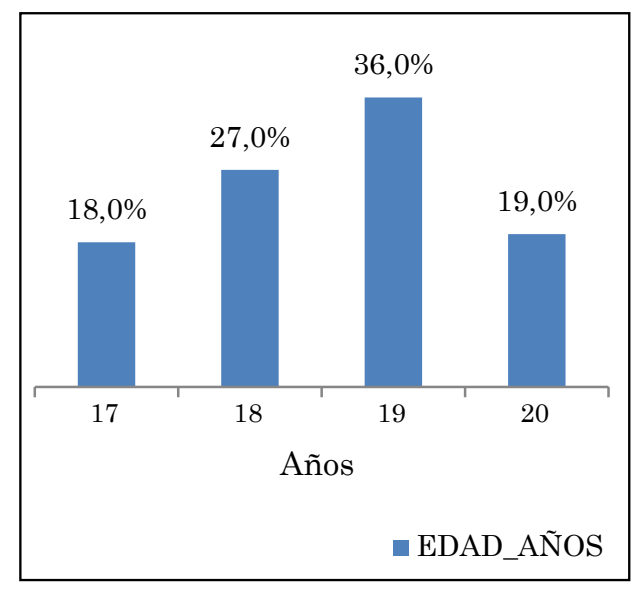

Figura 1. Medidas de tendencia central "EDAD"

Fuente: elaboración propia.

Fuente: elaboración propia. 


\section{Resultados}

Tal y como fue anunciado en el marco metodológico, inicialmente se presenta el análisis de frecuencias estadísticas por variable, con la intención de que el lector tenga un panorama ampliado de la participación de cada una dentro del estudio general.

Se encontró que la edad media de los encuestados es de 18,56 años, siendo 19 años la edad más frecuente (en 72 de las veces; par un $36 \%$ de los encuestados). El rango de edades alcanzó 4 valores distintos, con máximo de 20 años y mínimo de 17 años. Según se puede ver en la tabla 2 y su respectiva figura 1.

Resaltar que la población estudiada pertenece, mayoritariamente a estratos socioeconómicos bajos. Los resultados mostraron que alrededor de $81 \%$ de la muestra pertenece a estrado 2 o inferiores, y que, el estrato 1 es el de mayor frecuencia con $45,5 \%$. Por su parte, $46,5 \%$ eran hombres frente a la superior porción de mujeres, $53,5 \%$.

Respecto a la variable "Nivel de Educación Financiera", fue necesario darle tratamiento especial, toda vez que el instrumento utilizado arrojaba información de evaluación no homogénea. En ese sentido, se categorizó en tres niveles: ALTO, MEDIO y BAJO. En estos se representan tres intervalos de evaluación numérica, dada a cada respuesta en cada componente. Se dio valoración de 1 punto por respuesta favorable y 0 puntos para las respuesta no favorable. Desde esta óptica, el encuestado podría alcanzar entre 0 y 17 puntos. Sin embargo, cada componente constaba de diferente número de preguntas, por lo que fue indispensable estandarizar un valor de referencia que reflejara el punto de giro para categorizar si se tenía o no nivel de educación financiera favorable. Para ello, determinaron los puntos mínimos de favorabilidad por componente y luego se sumaron. Se tuvo que, los mínimos de favorabilidad eran 5,5 y 2 , para un total de 12 puntos como vértice de inflexión. Luego, para optimizar el rango medio (12 puntos) se consideró una desviación permisible de una respuesta favorable, es decir, 1 punto, el cual se acotó por encima y por debajo del vértice mínimo. Así las cosas, quienes alcanzaran puntuación en el intervalo cerrado $[11,13]$ tendrán un nivel de EF "MEDIA"; los ubicados en [0,10], "BAJA"; y los que caigan en [14,17], "ALTA". Respeto lo anterior, la tabla 3 y la figura 4, resumen las frecuencias observadas del nivel de EF de la muestra objeto. La mayoría de los encuestados tienen nivel "MEDIO" de EF, y cerca del $84 \%$ tienen nivel medio o bajo.

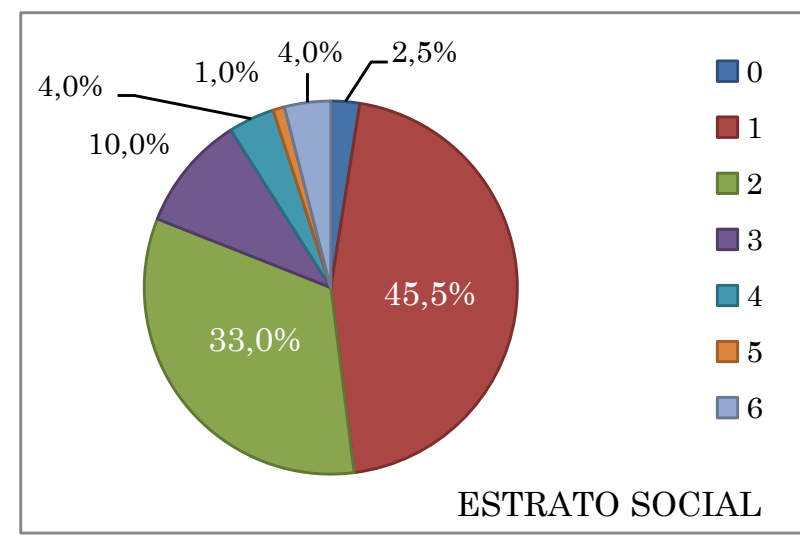

Figura 2. Estratificación de la muestra. Fuente: elaboración propia.

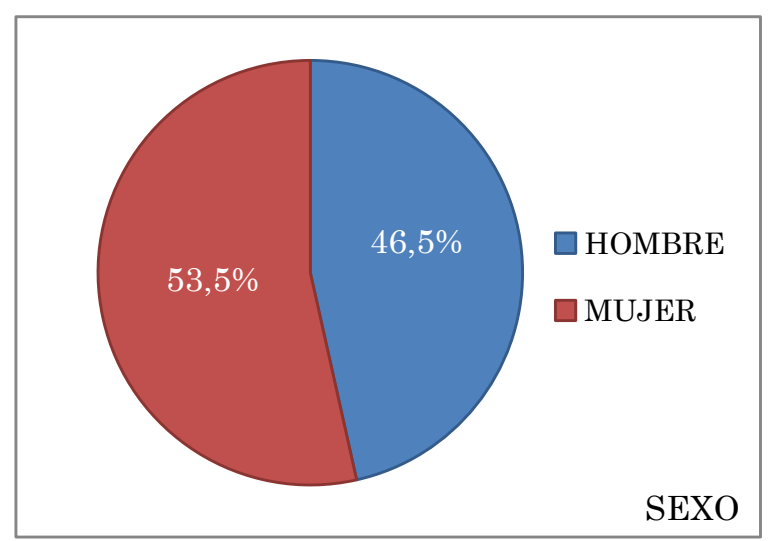

Figura 3. Distribución por sexo. Fuente: elaboración propia. 
Tabla 3

Nivel de EF según frecuencia observada

\begin{tabular}{lccc}
\hline $\begin{array}{l}\text { NIVEL } \\
\text { EDUFIN }\end{array}$ & $\begin{array}{c}\text { Frecuencia } \\
\text { absoluta (fi) }\end{array}$ & $\begin{array}{c}\text { Frecuencia } \\
\text { relativa (hi) }\end{array}$ & Porcentaje (\%) \\
\hline ALTO & 33 & 0,165 & $16,5 \%$ \\
MEDIO & 112 & 0,56 & $56,0 \%$ \\
BAJO & 55 & 0,275 & $27,5 \%$ \\
TOTAL & 200 & 1 & $100 \%$ \\
\hline
\end{tabular}

Fuente: elaboración propia.

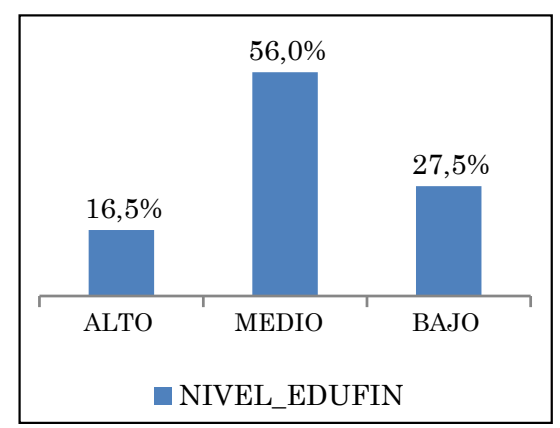

Figura 3. Frecuencia niveles de EF Fuente: elaboración propia.

\section{Inspección de las hipótesis}

La Hipótesis (" $H$ ") responde a la pregunta 1 ¿Existe relación significativa entre el nivel de EF y el programa académico estudiado? Con 18 grados de libertad, el test Chi cuadrado ( $X$ ${ }^{2}$ ) dio como resultado un $p$-valor observado de 0.009, inferior al alfa 0.05 máximo convenido. Cuando esto sucede, se rechaza la $H_{0}$ nula y se acepta la $H_{i}$ alternativa; por lo que se afirma: existe relación entre el nivel de EF calculado y el programa académico cursado. No obstante, se requiere inspección detallada de la relación intra categorías (programas), debido a que el estadístico Chi $\left(X^{2}\right)$ anota en los resultados que $33,3 \%$ de los Chi teóricos han esperado un recuento menor a 5 . Estoquiere decir que la relación entre variables podría deberse solo al concurso del $33,3 \%$ de las categorías estudiadas y el resto podrían no tener relación significativa. Para resolver la inquietud, se construyó la tabla de contingencias que contiene los $X^{2}$ calculados, que muestra la relación fuerte entre el nivel de EF del programa de Ingeniería Industrial en los niveles ALTO y BAJO, y Psicología para el nivel BAJO (tablas 4 y 5).

Tabla 4

Entrada de relación X2entre el nivel de EF y el programa académico cursado

\begin{tabular}{lccc}
\hline & Valor & gl & Significación asintótica (bilateral) \\
\hline Chi-cuadrado de Pearson & $35,261^{\mathrm{a}}$ & 18 &, 009 \\
Razón de verosimilitud & 38,023 & 18 &, 004 \\
N de casos válidos & 200 & & \\
\hline
\end{tabular}

a. 10 casillas $(33,3 \%)$ han esperado un recuento menor que 5. El recuento mínimo esperado es 3,30.

Fuente: Software SPSS con base en suministro de datos autónomos.

Tabla 5

Tabla de contingencia X2 y el programa académico cursado y nivel de EF

\begin{tabular}{cccccccccccc}
\hline $\begin{array}{c}\text { Nivel de } \\
\text { EF }\end{array}$ & DER. & PSICO. & $\begin{array}{c}\text { ING } \\
\text { SIS }\end{array}$ & \multirow{2}{*}{ ARQUI } & DEPORTE & $\begin{array}{c}\text { ING. } \\
\text { INDUS. }\end{array}$ & $\begin{array}{c}\text { TRAB } \\
\text { SOC. }\end{array}$ & ADMON & CONTA. & ECONO. & Totales \\
\hline ALTO & 0,512 & 0,512 & 0,148 & 1,603 & 0,512 & 6,694 & 0,512 & 0,876 & 0,876 & 0,512 & 12,758 \\
MEDIO & 0,004 & 1,575 & 0,004 & 1,289 & 0,914 & 0,057 & 0,057 & 0,129 & 0,004 & 1,289 & 5,321 \\
BAJO & 0,409 & 5,500 & 0,045 & 0,409 & 3,682 & 5,500 & 0,045 & 0,045 & 0,409 & 1,136 & 17,182 \\
Totales & 0,925 & 7,587 & 0,198 & 3,301 & 5,108 & 12,251 & 0,615 & 1,050 & 1,288 & 2,938 & 35,261 \\
\hline
\end{tabular}

Fuente: Software SPSS con base en suministro de datos autónomos. 
Económicas CUC 38: (2): Julio-Diciembre 2017, 101-111

Tabla 6

Tabla de contingencia X2 del programa académico cursado y nivel de EF

\begin{tabular}{lccc}
\hline & Valor & gl & Significación asintótica (bilateral) \\
\hline Chi-cuadrado de Pearson & $2,635^{\mathrm{a}}$ & 2 &, 268 \\
Razón de verosimilitud & 2,719 & 2 &, 257 \\
$\mathrm{~N}$ de casos válidos & 200 & & \\
\hline
\end{tabular}

a. 0 casillas $(0,0 \%)$ han esperado un recuento menor que 5 . El recuento mínimo esperado es 9,90.

Fuente: Software SPSS con base en suministro de datos autónomos.

A manera de contraste, esta información puede sustentarse al estudiar la relación entre los grupos de programas, según si pertenece a Ciencias Económicas o no. El resultado entonces es un $p$-valor de 0.268 con 2 grados de libertad. Esto se entiende como: un no existe relación significativa entre el nivel de EF y la ciencia asociada al programa, por lo que se reafirma la idea de una fuerte relación basada en el programa Ingeniería Industrial (Ciencia no económica) y no hacia el resto de programas (como lo detalla la tabla 6).
En adelante, los resultados demostraron la inexistencia de relación significativa entre el nivel de educación financiera de los estudiantes y las variables Edad, Estrato Social, y Sexo. Para todas, el cruce de frecuencias arrojó p-valores de $0.092,0.684$ y 0.876 , superiores al alfa máximo convenido, por lo que las hipótesis nulas $\mathrm{H}_{0}, \mathrm{H}_{0}, \mathrm{H} 4_{0}$, respectivamente, deben aceptarse; en consecuencia, descartar la posibilidad de relación entre las variables referenciadas por las mismas $H$, o lo que es igual, se rechazan las $H 2_{i}, H 3_{i}, H 4_{i}$ alternativas (ver tablas 7,8 y 9 ).

Tabla 7.

Tabla de contingencia X2 del nivel de EF y la edad del encuestado.

\begin{tabular}{lccc}
\hline & Valor & gl & Significación asintótica (bilateral) \\
\hline Chi-cuadrado de Pearson & $10,897^{\mathrm{a}}$ & 6 &, 092 \\
Razón de verosimilitud & 10,918 & 6 &, 091 \\
N. de casos válidos & 200 & & \\
\hline
\end{tabular}

a. 0 casillas $(0,0 \%)$ han esperado un recuento menor que 5 . El recuento mínimo esperado es 5,94.

Fuente: Software SPSS con base en suministro de datos autónomos.

Tabla 8

Tabla de contingencia X2 del nivel de EF y el estrato socio económico

\begin{tabular}{lccc}
\hline & Valor & gl & Significación asintótica (bilateral) \\
\hline Chi-cuadrado de Pearson & $9,221^{\mathrm{a}}$ & 12 &, 684 \\
Razón de verosimilitud & 13,017 & 12 &, 368 \\
N de casos válidos & 200 & & \\
\hline
\end{tabular}

a. 13 casillas $(61,9 \%)$ han esperado un recuento menor que 5. El recuento mínimo esperado es ,33.

Fuente: Software SPSS con base en suministro de datos autónomos. 
EDUCACIÓN FINANCIERA EN ESTUDIANTES UNIVERSITARIOS

Leonardo Beltrán Pinto - Efraín Gómez Martínez

Tabla 9

Tabla de contingencia X2 del nivel de EF y el sexo del encuestado.

\begin{tabular}{lccc}
\hline & Valor & gl & Significación asintótica (bilateral) \\
\hline Chi-cuadrado de Pearson &, $264^{\mathrm{a}}$ & 2 &, 876 \\
Razón de verosimilitud &, 264 & 2 &, 876 \\
N de casos válidos & 200 & & \\
\hline
\end{tabular}

a. 0 casillas $(0,0 \%)$ han esperado un recuento menor que 5 . El recuento mínimo esperado es 15,35.

Fuente: Software SPSS con base en suministro de datos autónomos.

Tabla 10

Tabla de contingencia X2 del nivel de EF y los grupos de estratos socio económicos.

\begin{tabular}{lccc}
\hline & Valor & gl & Significación asintótica (bilateral) \\
\hline Chi-cuadrado de Pearson & $7,763^{\text {a }}$ & 4 &, 101 \\
Razón de verosimilitud & 10,534 & 4 &, 032 \\
N de casos válidos & 200 & & \\
\hline
\end{tabular}

a. 3 casillas $(33,3 \%)$ han esperado un recuento menor que 5. El recuento mínimo esperado es 1,65.

Fuente: Software SPSS con base en suministro de datos autónomos.

Solo por dar valor a la alta porción de estudiantes que pertenecen a estaros 0,1 , y 2 (divisada en la figura 2), se realizó una inspección adicional a la $H 3$, reorganizando los estratos sociales en tres categorías: estratos bajos ( 0,1 y 2$)$, medios (3 y 4) y altos (5 y 6). La intención de esta recategorización fue determinar si, en lo particular, existía relación entre el nivel de EF y el tipo de sector socioeconómico de procedencia. Respecto lo anterior, el test $X^{2}$ reafirmó la no relación significativa entre las variables objetivo. El $p$-valor fue de 0.101 (Ver tabla 10).

\section{Conclusiones}

Se inició este articulo enumerando las consecuencias que tendría para la economía colombiana el analfabetismo financiero, a través del marco referencial se pudo identificar subgrupos más vulnerables. Estos Subgrupos (sexo, edad y nivel socioeconómico) fueron identificados dentro de la Corporación Universitaria del Caribe (CECAR) en una población de delimitada en los semestres del quinto (5)-Sexto (6). Permitiendo así que las competencias no adquiridas dentro de los colegios de la región, pudiese complementarse con la formación financiera de la institución.

Los resultados demostraron que no existe relación significativa entre el nivel de educación financiera evaluado en la muestra de estudiantes, frente a variables específicas como el Programa Estudiado, el Sexo o el Estrato socio económico de procedencia. Tampoco se puede afirmar que exista 
concordancia o relación entre estudiar un programa asociado o no a las ciencias económicas y el nivel de educación financiera. No obstante, si se encontraron conexiones significativas con la edad del encuestado, pudiéndose concluir que resulta más probable que el nivel de educación financiera se asocia a la edad de un individuo que a su formación profesional.

De igual forma, el presente estudio también buscaba condiciones exploratorias sobre el nivel de educación financiera de adultos y en ningún caso, se pretendió desarrollar afirmaciones inferenciales y/o proyectivas a la población estudiantil. Se hace fundamental avanzar en el estudio con futuras mediciones que abarquen mayor cantidad de sujetos y componentes en el instrumento de medición.

\section{Referencias}

ASOBANCARIA. (2014). Comienza la educación financiera en los colegios. 18 De Julio De 2014. Recuperado de https://www.larepublica.co/ asuntos-legales/opinion/comienzala-educacion-financiera-en-los-colegios- 2147161

Bernheim, B. D., \& Scholz, J. K. (1993). Private Saving and Public Policy. Tax Policy and the Economy (Vol. 7). https://doi.org/10.2307/20060630

Denegrí, M., Del Valle, C., González, Y., Etchebar-ne, S., Sepúlveda, J., \& Sandoval, D. (2014). ¿Consumidores o ciudadanos?: Una propuesta de inserción de la educación económica y financiera en la formación inicial docente. Estudios Pedagógicos (Valdivia), 40(1), 75-96. https://doi.org/10.4067/ S0718-07052014000100005
Gerardi, K., Götte, L., Meier, S., Goette, L., \& Meier, S. (2010). Financial Literacy and Subprime Mortgage Delinquency: Evidence from a Survey Matched to Administrative Data. Federal Reserve Bank of Atlanta Working Paper Series No. 2010-10 https:// doi.org/10.2139/ssrn.1600905

Hernández, R., Fernández C., y Baptiste, P. (2014). Metodología de la investigación. México, D.F.: McGraw-Hill.

Klot, S., Peters, A., Aalto, P., Bellander, T., Berglind, N., D'Ippoliti, Elosua, R., Hörmann, A., Kulmala, M., Lanki, T., Löwel, H., Pekkanen, J., Picciotto, S. Sunyer, J. \& Forastiere, F. (2005). Ambient Air Pollution Is Associated With Increased Risk of Hospital Cardiac Readmissions of Myocardial Infarction Survivors in Five European Cities. Circulation, 112(20), 3073-3079. https://doi.org/10.1161/ CIRCULATIONAHA.105.548743

Lusardi, A. \& Mitchell, O. S. (2011). Financial literacy around the world: An overview. Journal of Pension Economics and Finance, 10(4), 497-508. https://doi.org/10.1017/ S1474747211000448

Lusardi, A., \& Tufano, P. (2015). Debt literacy, financial experiences, and overindebtedness. Journal of Pension Economics and Finance, 14(4), 332-368. https://doi.org/10.1017/ S1474747215000232

OCDE (2005a). Improving Financial Literacy. Financial Market Trends, 2005(2), 111-123. https://doi.org/10.1787/fmtv2005-art11-en

OCDE (2005b). Improving Financial Literacy. OECD Publishing. https://doi. org/10.1787/9789264012578-en 
EDUCACIÓN FINANCIERA EN ESTUDIANTES UNIVERSITARIOS

Leonardo Beltrán Pinto - Efraín Gómez Martínez

República de Colombia. Congreso de la República. (15 de julio de 2009) Por la cual se dictan normas en materia financiera, de seguros, del mercado de valores $y$ otras disposiciones. [LEY 1328] DO: 47411. Recuperado de http://www.secretariasenado.gov.co/senado/basedoc/ ley_1328_2009.html 